\title{
Oral Histoplasmosis
}

\author{
Gillian A. Folk ${ }^{1,2}$ • Brenda L. Nelson ${ }^{1,2,3}$
}

Received: 10 January 2017 / Accepted: 3 February 2017 / Published online: 20 February 2017

(C) Springer Science+Business Media New York (outside the USA) 2017

\begin{abstract}
A 44-year-old female presented to her general dentist with the chief complaint of a painful mouth sore of 2 weeks duration. Clinical examination revealed an irregularly shaped ulcer of the buccal and lingual attached gingiva of the anterior mandible. A biopsy was performed and microscopic evaluation revealed histoplasmosis. Histoplasmosis, caused by Histoplasma capsulate, is the most common fungal infection in the United States. Oral lesions of histoplasmosis are generally associated with the disseminated form of histoplasmosis and may present as a fungating or ulcerative lesion of the oral mucosa. The histologic findings and differential diagnosis for oral histoplasmosis are discussed.
\end{abstract}

Keywords Histoplasmosis · Fungi · Oral ulcer · Lupus erythematosus $\cdot$ Histoplasma capsulatum $\cdot$ Antimycotic

Special thank you to Dr. Moris Aynechi of Bakersfield, California for contributing the clinical photos.

Disclaimer The opinions and assertions expressed herein are those of the author and are not to be construed as official or representing the views of the Department of the Navy or the Department of Defense.

Brenda L. Nelson

brenda.1.nelson24.mil@mail.mil

1 Academy of Our Lady of Peace, San Diego, CA, USA

2 Department of Head and Neck Pathology, NMCSD, San Diego, CA, USA

3 Department of Anatomic Pathology, Naval Medical Center San Diego, 34800 Bob Wilson Drive, San Diego, CA 92134-5000, USA

\section{History}

A 44-year-old female presented to her general dentist with the chief complaint of a painful mouth sore of 2 weeks duration. Clinical examination revealed an irregularly shaped ulcer of the buccal and lingual attached gingiva of the anterior mandible. The patient was asked to return in 2 weeks for re-evaluation, and the lesion persisted. At the 2 week follow-up appointment, the gingival ulcer had increased in size and coalescing ulcers involving the entire mandibular buccal vestibule mucosa were also present. The ulcers were irregularly shaped and demonstrated a white surface and focally rolled, raised borders (Figs. 1, 2). A thorough head and neck exam resulted in the detection of mild bilateral lymphadenopathy of the neck. The patient's medical history was significant for systemic lupus erythematosus, for which she is under regular care of her physician. Her medications include Plaquenil, Prednisone, Azathioprine and Warfarin, all prescribed for treatment of her lupus. No travel history outside the United States was reported. The clinical differential diagnosis for the oral lesions included infectious diseases, lupus erythematous, erosive lichen plans, and squamous cell carcinoma. A biopsy of the anterior mandibular vestibule mucosa was performed and submitted to pathology.

\section{Diagnosis}

Histologic examination of hematoxylin and eosin stained slides show a mucosal surface that is partially ulcerated and covered by a fibrinopurulent membrane. The specimen is notable for a dense inflammatory infiltrate consisting of prominent histiocytes, lymphocytes and plasma cells. Innumerable $2-4 \mu \mathrm{m}$ round to oval, narrow-based 


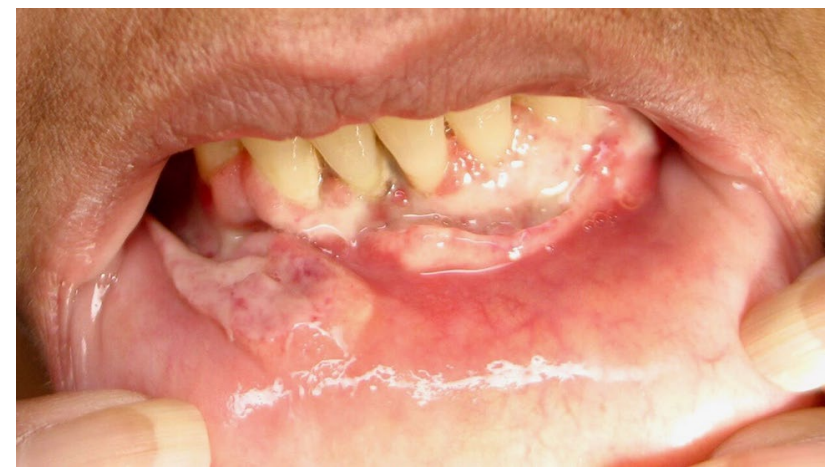

Fig. 1 Clinical photograph showing multiple ulcers on anterior mandibular facial gingiva and a fungating ulcerative mass with rolled borders on lower labial mucosa

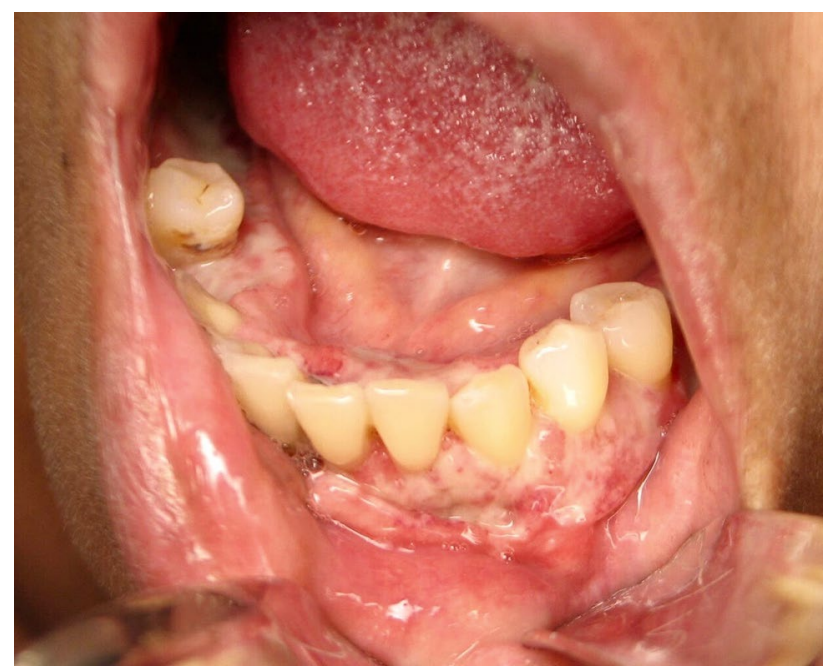

Fig. 2 Diffuse ulcers involving bilateral mandibular gingiva (facial and lingual) and vestibular mucosa

budding amphophilic spherules surrounded by a peripheral pale zone (halo) are readily identified within the cytoplasm of the histiocytes (Fig. 3). Special stains Grocott-Gomori methenamine silver (GMS) and periodic acid-Schiff (PAS) were reactive and highlight fungal organisms (Figs. 4, 5, 6). The fulminant histologic presentation was immediately and highly suggestive of histoplasmosis. The case was diagnosed as consistent with histoplasmosis with a comment recommending serologic testing. The patient's clinical presentation in conjunction with her history of immunosuppression resulted in the diagnosis of disseminated histoplasmosis by her primary care physician. An infectious disease specialist was consulted. Details of the patient's treatment were not available; however, the patient was described as recovered from her infection.

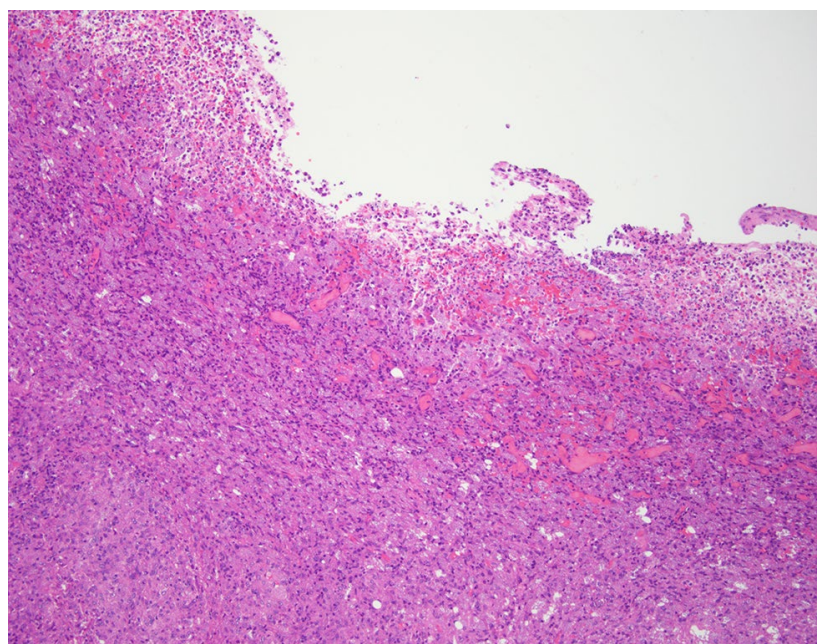

Fig. 3 Low power hematoxylin and eosin demonstrates the dense inflammatory infiltrate

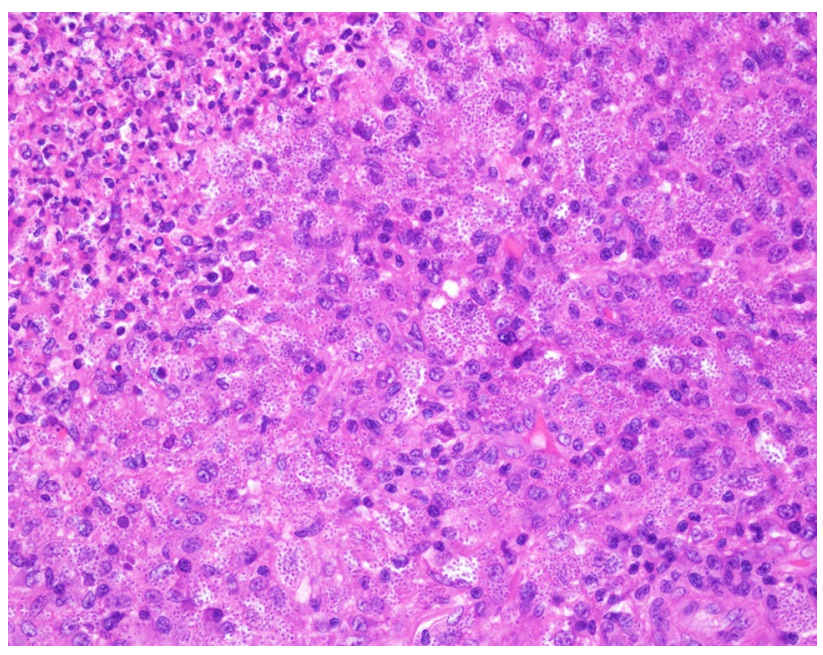

Fig. 4 High power hematoxylin and eosin shows numerous 2-4 $\mu \mathrm{m}$ yeast of $H$. capsulatum

\section{Discussion}

Histoplasmosis, a disease caused by the dimorphic fungus Histoplasma capsulatum, is the most common systemic fungal infection in the United States. Dimorphic fungi manifest in mold form in soils while at ambient temperatures, while taking on a yeast form when infecting a human host. Inhalation of airborne spores is generally the means of transmission. The rich soils of the Ohio and Mississippi rivers valleys are endemic with the fungi. Soils contaminated by bat and bird guano are considered to be an environment that is particularly high risk for exposure and infection, especially if the ground cover is disrupted due to construction or nature processes. H. capsulatum is not limited to the United States can be found 


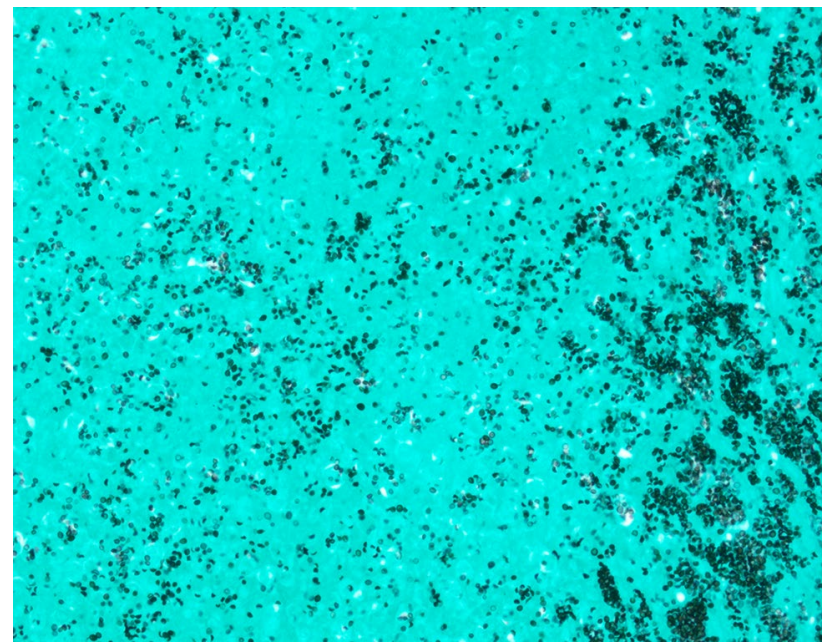

Fig. 5 Grocott-Gomori methenamine silver reaction (GMS) highlights the fungal organisms

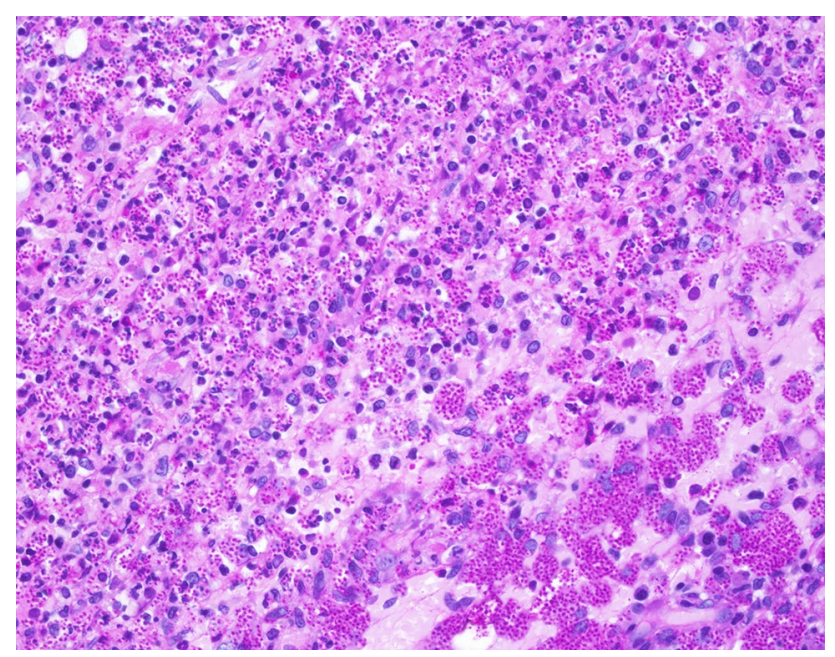

Fig. 6 Periodic acid-Schiff (PAS) is also useful in visualizing the innumerable fungal elements

in parts of Central and South America, Africa, Asia, and Australia.

For those who contract histoplasmosis, symptoms may occur in as little as a few days or as many as few weeks. Most affected individuals, especially those immunocompetent, show no signs or symptoms of infection. The severity of the disease may be related to the burden of spores inhaled, with high exposure rates resulting in a more dramatic presentation. Histoplasmosis occurs in three forms: acute, chronic, and disseminated. Acute histoplasmosis is characterized by a self-limiting pulmonary infection that causes symptoms similar to that of influenza. Chronic histoplasmosis may appear clinically similar to tuberculosis, affecting primarily the lungs and those affected are often immunosuppressed. The disseminated form, also affects disproportionally the immunocompromised, and may affect multiple organ systems: spleen, adrenal glands, liver, lymph nodes, gastrointestinal tract, central nervous system, kidneys, and oral mucosa.

The oral lesions of disseminated histoplasmosis can occur at any oral mucosa, but are most commonly found on the tongue, palate, and buccal mucosa. Patients may present with painful ulcers that persist for several weeks. The clinical appearance may be similar to that of a malignancy, prompting the clinician to biopsy the lesion.

Histologic findings of histoplasmosis typically show diffuse lymphohistiocytic infiltrates with fungal elements about $2-4 \mu \mathrm{m}$ in size detected within the cytoplasm of histiocytes. Well-formed granulomas are generally rare. Special stains, such as GMS and PAS methods will highlight the fungal organisms' cell wall. A classic "halo" appearance caused by the cytoplasm retracting from the thick cell wall is helpful in identifying the fungi. Additionally, the diagnosis may be assisted via laboratory tests; however, sensitivity may be variable especially in the disseminated form of histoplasmosis.

The clinical differential diagnosis of a lesion of oral histoplasmosis is broad. Benign entities ranging from nonspecific ulcers to malignancy are considerations. The histologic differential diagnosis narrows with the presence of a dense inflammatory infiltrate, and a reactive or infectious process is generally rapidly considered. Infectious lesions with varied etiologies may be considered: bacterial, viral, parasitic or fungal. Bacterial infections include sexual transmitted infections (Neisseria gonorrhea and Treponema pallidum) and may need to be excluded by utilizing immunohistochemical stains. Viral infections such as herpes simplex type I or II are to be considered, but generally demonstrate classic viral cytopathic affect, known as ballooning degeneration. Parasitic infections, such as leishmaniasis, may be rare in some parts of the world, but travel history should be investigated to aid in excluding this and other unusual infections. Other fungal diseases such as blastomycosis and cryptococcosis also need to be considered. Blastomycosis is generally described as a larger organism, 8-20 $\mu \mathrm{m}$, with broad attachment between cells. In contrast, cryptococcosis is 4-6 $\mu \mathrm{m}$ with a thick capsule that appears like a halo on microscopy. The use of special stains is helpful in the analysis of size and shape of the individual fungal elements, and is imperative in determining the particular fungal species. Specimens for culture are always a consideration as well as expert consultation, to include that of an infectious disease specialist.

In some patients, histoplasmosis will resolve without intervention. However, those with oral lesions generally need comprehensive treatment. The severity of the infection and the patient's underlying health problems will often direct treatment, and antimycotic drugs are generally 
indicated. These medications frequently result in numerous and serious side effects, compounding the difficulty in treating these patients. Prognosis is, in turn, directly related to the severity and health status of the patient and can be variable.

\section{References}

1. Viswanathan S, Chawla N, D'Cruz A, Kane SV. Head and neck histoplasmosis-a nightmare for clinicians and pathologists! Experience at a Tertiary Referral Cancer Centre. Head Neck Pathol. 2007;1(2):169-72. doi:10.1007/s12105-007-0034-1.

2. Vidyanath S, Shameena PM, Sudha S, Resmi G Nair. Disseminated histoplasmosis with oral and cutaneous manifestations. J Oral Maxillofac Pathol. 2013;17(1):139-42. doi:10.4103/0973-029X.110722.
3. Patil K, Mahima VG, Prathibha Rani RM. Oral histoplasmosis. J Indian Soc Periodontol. 2009;13(3):157-9. doi:10.4103/0972-124X.60230.

4. Brazão-Silva MT, Mancusi GW, Bazzoun FV, Ishisaki GY, Marcucci M. A gingival manifestation of histoplasmosis leading diagnosis. Contemp Clin Dent. 2013;4(1):97-101. doi:10.4103/0976-237X.111621.

5. Mohammed S, Sinha M, Chavan P, Premalata CS, Shivaprakash MR, Chakrabarti A, Jayshree RS. Oral histoplasmosis masquerading as oral cancer in HIV-infected patient: a case report. Med Mycol Case Rep. 2012;1(1):85-7. doi:10.1016/j. mmcr.2012.09.002.

6. Akin L, Herford AS, Cicciù M. Oral presentation of disseminated histoplasmosis: a case report and literature review. J Oral Maxillofac Surg. 2011;69(2):535-41. doi:10.1016/j. joms.2010.05.053.

7. O'Sullivan MV, Whitby M, Chahoud C, Miller SM. Histoplasmosis in Australia: a report of a case with a review of the literature. Aust Dent J. 2004;49(2):94-7. 